\title{
Comparison of three primitive reflexes in neurological patients and in normal individuals
}

\author{
JPA JENSEN, U GR ØN, H PAKKENBERG \\ From the Department of Neurology, Hvidovre University Hospital, Hvidovre, Denmark
}

SUMmARY Two hundred and sixty-five normal individuals and patients with clinical signs of extracranial disease, 174 patients with intracranial disease without signs of basal ganglia involvement and 126 patients with signs of basal ganglia disorders were examined clinically for the occurrence of the activation phenomenon of rigidity, the palmomental reflex and the glabella tap response. A comparison between the three groups, decade for decade, showed that the activation phenomenon was so common in the first two groups that its presence was unlikely to be the first sign of Parkinson's disease. A tendency to increasing frequency with increasing age was noted. The palmomental reflex and glabella tap response were more frequent in the last group, but also so common in the first two, that the clinical significance was marginal. No tendency to increasing occurrence with increasing age was noted.

The significance of certain easily elicited signs has been stressed as early evidence of Parkinson's disease by several authors. Thus Pearce et al mention that the palmomental reflex and Dalby ${ }^{2}$ that an exaggerated palmomental reflex can be the first sign of Parkinson's disease. Garland ${ }^{3}$ and Pearce et al lay similar significance on the glabella tap response (nasopalpebral reflex, orbicularis reflex, blinking reflex, Myerson's sign). Webster describes the activation phenomenon not only as an early sign, but also uses a positive reaction to discern between different degrees of rigidity. This might lead to a higher or lower score on the Webster rating scale or in misinterpretation of a physiological tremor. In later years, however, other authors ${ }^{5}$ have found these parameters to be of minor relevance, though there is no clear-cut uniform agreement.

The purpose of this investigation was to observe the frequency of the activation phenomenon, the palmomental reflex and the glabella tap response in different groups of patients and in normal individuals and to observe any clinical differences between these groups and patients with Parkinson's disease or other basal ganglia disorders.

Address for reprint requests: Dr John Jensen, Department for Epileptics, Filadelfia, Dianalund, 4293 Denmark.

Received 24 June 1982 and in revised form 2 October 1982 Accepted 18 October 1982

\section{Methods and material}

During the years 1977-1979 the authors examined 573 patients, age limits $19-87$ years. The clinical neurological examination of referred patients (both hospitalised and outpatients) routinely included the activation phenomenon, the palmomental reflex and the glabella tap response. A normal population with no history or signs of neurological disease (relatives, ward-staff, colleagues etc) was included and examined for these phenomena. Case history included information of traits to hereditary tremor, past history of neurological diseases, present symptoms of tremor, rigidity, akinesia and dyskinesia and medication, stressing those drugs which are known to or supposed to act on the basal ganglia.

The activation phenomenon was elicited in accordance with Webster's description. Slow and rapid passive movements of the upper extremities as in a routine examination for tone were noted with the contralateral arm at rest and with the contralateral arm extended, the patient being then asked to draw circles in the air with the fingers of the contralateral arm. If this led to a marked increase of tone in the passively moved arm, it was registered as a positive activation phenomenon.

The palmomental reflex was elicited as described by Dalby ${ }^{2}$ and Jacobs and Gossman. ${ }^{7}$ During a subnoxious stroking of the thenar eminence with a blunt instrument a puckering of the ipsilateral mentalis was registered as a positive palmomental reflex.

We elicited the glabella tap, described by various authors ${ }^{18}$ in the following manner. While the patient looked straight ahead, the examiner approached his index finger from above (outside the visual field, so as to avoid a startle reaction) and tapped the glabella with a frequency of about 
two per second. Blinking that ceased after a few taps was disregarded. A continuous synchronous blinking of the eyelids, especially the lower eyelids, was registered as a positive response. A positive response was evaluated as grade 1 if the blinking followed tapping of the glabella region alone, grade 2 if blinking followed tapping of the lower forehead and grade 3 if blinking followed tapping of the upper forehead near the hair limit. If no description of this grading was given, the glabella tap response was registered as "non-specified". All relevant data were registered on special questionnaires printed for this study. After excluding eight patients due to insufficient data, the population, totalling 565, 247 males, 318 females, was divided into five subgroups according to the clinical diagnosis.

(1) normal, total 72 , age limit 19-76,

(2) extracranial diseases, total 193, age limit 19-81, diagnoses are shown in table 1 ,

(3) intracranial disorders with no clinical evidence of involvement of the basal ganglia, total 174, age limit 2089 , the clinical, pathological diagnoses are shown in table 2 . Only in a minority would a more exact anatomical localisation be possible and has not been attempted,

(4) Parkinson's disease including five cases of drug-induced Parkinsonism, total 87 , age limit 41-84. Five of the cases were drug-induced, six presumably drug-induced (by various neuroleptics and metoclopramide, being only treated with anticholinergic drugs). Twenty-six other cases received no medication at the time of investigation. Of the remaining 50 cases, 32 were on monotherapy with either Sinemet or Madopar (21), anticholinergics (4), amantadine (3), bromocriptine (2), levodopa (1) or propranolol (1). Eighteen received polytherapy consisting of a combination of Sinemet or Madopar and anticholinergics (12), levodopa and anticholinergics (3) and bromocriptine and anticholinergics (3). As the patients were only seen once and at different stages of the disease, the dosages vary,

(5) other basal ganglia disorders, total 39, age limit 30-86, diagnoses are shown in table 3 .

These five subgroups were collected in three major groups. I (subgroups 1 and 2) constituted our normal population, II (subgroup 3) intracranial disease without involvement of basal ganglia and III (subgroups 4 and 5) basal ganglia disorders. Totals were 265, 174 and 126 respectively. The three groups were compared decade for decade. Percentages are only given in groups of more than

\section{Table 1 Extracranial diseases}

Muscular pains (incl. tension headache)

Psychogenic disturbanices

Peripheral nerve lesions

Disturbances in loco-motor system

Odontogenic disease

Facial pain

Cardiopathy, endocrine disorders etc.

Myelopathy

No signs of organic nerve disease

Total

\begin{tabular}{l}
55 \\
38 \\
$25^{*}$ \\
24 \\
11 \\
$9^{*}$ \\
9 \\
3 \\
19 \\
\hline 193
\end{tabular}

${ }^{*}$ No sensory loss demonstrated.
Table 2 Intracranial disorders with obvious or possible involvement of the brain, but probably not of the basal ganglia

\begin{tabular}{lr}
\hline Migraine & 46 \\
Postural hypotension, fainting spells & 21 \\
Stroke & 19 \\
Brain atrophy & 17 \\
Epilepsy & 14 \\
Brain trauma & 12 \\
Transitory ischaemic attacks & 11 \\
Brain tumour & 8 \\
Multiple sclerosis & 8 \\
Arterial hypertension & 8 \\
Vertigo & 7 \\
Cerebral palsy & 3 \\
Total & 174 \\
\hline
\end{tabular}

Table 3 Basal ganglia disorders or diseases with evident or possible involvement of the basal ganglia

\begin{tabular}{lr}
\hline Parkinsonism (5 drug induced) & 87 \\
Essential and senile tremor & 26 \\
Dyskinesias & 13 \\
BLM-syndrome & 4 \\
Torticollis & 3 \\
Chorea Huntington & 3 \\
Unclassified dyskinesia & 2 \\
Athetosis & 1 \\
Total & 126 \\
\hline
\end{tabular}

15 individuals. Statistical analysis was done with Fischer's exact test ( $\chi^{2}$ test using Yates correction for continuity).

\section{Results}

\section{Activation phenomenon}

The comparison between activation phenomenon in the normal population and in patients without and with involvement of the basal ganglia is shown in table 4 . In the first two groups a tendency towards increasing frequency with increasing age is noted. There is no obvious corresponding trend in the third group (disorders of the basal ganglia), but the total numbers are small. A significant difference in occurrence of the activation phenomenon is noted between the first and second groups compared with the third $(52,42$ and $14 \%$ respectively) $(2 \mathrm{p}<0.0001)$.

\section{Palmomental reflex}

The comparison between the three groups as outlined above is shown in table 5. No obvious trend towards increasing occurrence with increasing age is evident in the first two groups. A significant difference in occurrence of the palmomental reflex is noted between the first two groups and the third (11, 12 and $24 \%$ respectively) $(2 p=0 \cdot 029)$. 
Table 4 Comparison of incidence of activation-phenomenon in the three groups, decade for decade. Group I, II, and III: see text

\begin{tabular}{|c|c|c|c|c|c|c|c|c|c|c|}
\hline & & 2 Decade & 3 Decade & 4 Decade & 5 Decade & 6 Decade & 7 Decade & 8 Decade & 9 Decade & Total \\
\hline \multirow{2}{*}{ Group I } & No patients & 4 & 53 & 66 & 39 & 48 & 33 & 18 & 4 & 265 \\
\hline & Activation & 1 & $16(30 \%)$ & $26(40 \%)$ & $22(56 \%)$ & $29(60 \%)$ & $29(88 \%)$ & 10 & 3 & $136(52 \%)$ \\
\hline \multirow{2}{*}{ Group II } & No patients & & 27 & 29 & 23 & 32 & 41 & 16 & 6 & 174 \\
\hline & Activation & & 6 & 4 & 12 & $21(66 \%)$ & $22(54 \%)$ & 7 & 1 & $73(42 \%)$ \\
\hline \multirow{2}{*}{ Group III } & No patients & & & 4 & 6 & 25 & 45 & 34 & 12 & 126 \\
\hline & Activation & & & & & 3 & 6 & 5 & 4 & $18(14 \%)^{*}$ \\
\hline
\end{tabular}

*Different from group I and II $(2 \mathrm{p}<0 \cdot 0001)$.

Table 5 Comparison of incidence of palmomental reflex in the three groups, decade for decade

\begin{tabular}{|c|c|c|c|c|c|c|c|c|c|c|}
\hline & & 2 Decade & 3 Decade & 4 Decade & 5 Decade & 6 Decade & 7 Decade & 8 Decade & 9 Decade & Total \\
\hline \multirow{2}{*}{ Group I } & No patients & 4 & 53 & 66 & 39 & 48 & 33 & 18 & 4 & 265 \\
\hline & Palmomental & & 1 & 7 & 7 & 7 & 5 & 3 & & $30(11 \%)$ \\
\hline \multirow{2}{*}{ Group II } & No patients & & 27 & 29 & 23 & 32 & 41 & 16 & 6 & 174 \\
\hline & Palmomental & & 5 & 2 & 3 & 1 & 7 & 2 & 0 & $20(11 \cdot 5 \%)$ \\
\hline \multirow{2}{*}{ Group III } & No patients & & & 4 & 6 & 25 & 45 & 34 & 12 & 126 \\
\hline & Palmomental & & & 1 & & 2 & $12 \dagger$ & $11 \dagger$ & $4 \dagger$ & $30(24 \%)^{*}$ \\
\hline
\end{tabular}

${ }^{*}$ Different from group I and II $(2 \mathrm{p}=0.029)$.

†All Parkinson's disease.

Table 6 Comparison of incidence of glabella tap response in the three groups, decade for decade

\begin{tabular}{|c|c|c|c|c|c|c|c|c|c|c|}
\hline & & 2 Decade & 3 Decade & 4 Decade & 5 Decade & 6 Decade & 7 Decade & 8 Decade & 9 Decade & Total \\
\hline \multirow{2}{*}{ Group I } & No patients & 4 & 53 & 66 & 39 & 48 & 33 & 18 & 4 & 265 \\
\hline & Glabella tap & 1 & $17(32 \%)$ & $23(35 \%)$ & $15(38 \%)$ & $17(35 \%)$ & 14 & 7 & 1 & $95(36 \%)$ \\
\hline \multirow{2}{*}{ Group II } & No patients & & 27 & 29 & 23 & 32 & 41 & 16 & 6 & 174 \\
\hline & Glabella tap & & 9 & 8 & 8 & 7 & $16(39 \%)$ & 7 & 6 & $61(35 \%)$ \\
\hline \multirow{2}{*}{ Group III } & No patients & & & 4 & 6 & 25 & 45 & 34 & 12 & 126 \\
\hline & Glabella tap & & & 4 & 4 & $20(80 \%)$ & $31(69 \%)$ & $23(68 \%)$ & 8 & $90(71 \%)^{*}$ \\
\hline
\end{tabular}

${ }^{*}$ Different from group I and II $(2 p<0 \cdot 0001)$.

Table 7 Comparison of the three reflexes in the three groups (see text)

\begin{tabular}{|c|c|c|c|c|}
\hline & & \multicolumn{3}{|l|}{ Results } \\
\hline & & Group I & Group II & Group III \\
\hline $\begin{array}{l}\text { Activation } \\
\text { Palmomental } \\
\text { Glabella tap }\end{array}$ & & $\begin{array}{l}52 \% \\
11 \% \\
36 \%\end{array}$ & $\begin{array}{l}42 \% \\
12 \% \\
35 \%\end{array}$ & $\begin{array}{l}14 \% \\
24 \% \\
71 \%\end{array}$ \\
\hline \multirow[t]{2}{*}{$\begin{array}{l}\text { Glabella tap } \\
\text { Non-specified }\end{array}$} & $\begin{array}{l}+ \\
++ \\
++\end{array}$ & $\begin{array}{r}73 \% \\
20 \% \\
2 \% \\
5 \%\end{array}$ & $\begin{array}{r}67 \% \\
23 \% \\
3 \% \\
7 \%\end{array}$ & $\begin{array}{r}37 \% \\
40 \% \\
17 \% \\
6 \%\end{array}$ \\
\hline & & $100 \%$ & $100 \%$ & $100 \%$ \\
\hline
\end{tabular}

Glabella tap response

The comparison between the three groups is shown in table 6 . There is no clear-cut evidence of increasing occurrence with increasing age in any group. There is a significant difference between occurrence in the first two groups and the third $(36,35$ and $71 \%$ respectively) $(2 p<0.0001)$.

Comparison of the graded evaluation (table 7) shows that the majority of patients in the first two groups have a grade 1 response (that is a slight reaction) and that the majority of patients in group III have a grade 2 response (that is a more pronounced reaction). 
Finally, although the total numbers were small, we compared the two subgroups 4 and 5, of which our basal ganglia disorder group III consisted. As for the activation phenomenon, no significant difference was evident ( $p>0 \cdot 1)$, but on comparing the palmomental reflex and glabella tap response, a significant difference was noted $(\mathrm{p}<0.001$ and $\mathrm{p}<0.05$ respectively).

\section{Discussion}

\section{Activation phenomenon}

The inability to maintain two voluntary motor activities at the same time is a common observation in the daily life of patients with Parkinson's disease (for example shaving, dressing) and may be more responsible for their slowness than rigidity per se. To our knowledge, however, little description of this phenomenon has been given in the literature. Increase of rigidity on activation was originally described by Berkwitz ${ }^{9}$ and found present in 10 normal subjects. Webster ${ }^{10}$ describes a dynamic study of Parkinson patients and six normal subjects, none of the latter showed response to activation (following a beam of a flash-light with the non-examined arm). Schwab et $a l^{11}$ describe a quantitative test comparing ergographic curves of 150 patients with Parkinsonism and 50 normal subjects. No total numbers are given. A tendency to impaired functioning in the test begins to show in the normal subjects in the seventh and eighth decade, which the authors regard as a normal ageing process. However, the differences in Parkinsonism patients of the age group is four to five times greater. Pearce ${ }^{12}$ describes paratonia and Paulson and Gottlieb ${ }^{13}$ gegenhalten as having common features with the activation phenomenon and as a rather non-specific sign in elderly and demented patients.

We have not been able to demonstrate a clinical difference in our groups suggesting an increased incidence in basal ganglia affection nor a significant difference between the two basal ganglia disorder subgroups. On the contrary, we were surprised to find how frequent the activation phenomenon was in our normal group. The lower incidence in Parkinsonism could be explained by the definition of a positive finding being an evident and marked increase in tone on activation. Thus only nine patients out of 87 with Parkinsonism showed the classical activation phenomenon. We found that on activation 70 out of 87 patients with Parkinsonism had only a minor increase of hypertonia already present and these were not registered as positive activation phenomenon. The very high incidence of approximately $50 \%$ in persons without signs of intracranial disease and $40 \%$ in persons without signs of basal ganglia disease cannot support a theory that this phenomenon can be attributed to involvment of the basal ganglia, and hardly any significance can be laid in its being present or absent.

\section{Palmomental reflex}

The incidence of this reflex, first described some 60 years ago in normal individuals, varies in different investigations from approximately $2-60 \%$ (review $\mathrm{see}^{6}$ ). $\operatorname{Reis}^{14}$ found a mentalis contraction in all subjects studied electromyographically, provided the stimulus was strong enough, considering the reflex to be only a regional variant of a generalised reaction to nociceptive stimuli. Others ${ }^{13}$ feel, on the basis of its common occurrence in infants, that its presence in adults might reflect a "release phenomenon". As to increasing occurrence with increasing age there is also some disagreement, Blake and Kunkle ${ }^{6}$ stating no differences to be found and Jacobs and Gossman 7 demonstrating an increase in incidence comparing normal individuals from $20 \%$ in the fifth decade to $60 \%$ in the ninth decade. Neither is there any agreement as to its incidence in pathological cases, although most ${ }^{2}{ }^{5}{ }^{13}$ agree on an increased incidence in disorders of the upper motor neurone. Gossman and $\mathrm{Jacobs}^{5}$ attributed the incidence of $48 \%$ in 58 Parkinsonism patients to ageing rather than to the disease, finding no difference in various degrees of severity. Blake and Kunkle ${ }^{6}$ and Dalby ${ }^{2}$ stress the importance of discerning between a "normal" reflex defined as a small, shortlasting contraction of the mentalis muscle only elicitable from the thenar region with rapid habituation, and a "pathological" response with less habituation and a larger reflexogenic zone, the latter being a justifiable alerting sign of upper motor neurone disorder. Klawans and Paulson ${ }^{15}$ mention that the reflex is not reversed by levodopa. They find no foundation for the concept that a palmomental reflex can be useful in identifying Parkinsonism in patients with cortical atrophy and dementia.

Our results differ in many respects from other observations. We did not find any tendency towards increasing frequency with age. The incidences we found in normal individuals is not very high. This could be explained by our stimulus not being of sufficient strength. However this would fail to explain why a correspondingly low incidence in suprasegmentary lesions was found, where a pathological or easily elicited response was to be expected. ${ }^{2}$ Our findings of increased incidence in the group with basal ganglia disorders are also in contrast with the finding of Gossman and Jacobs. ${ }^{5}$ On comparing the two subgroups 29 of the 30 positive findings were in the Parkinsonism subgroup. When comparing the three groups, however, the relatively small differences would support Reis' statement ${ }^{14}$ that this reflex has limited clinical value. 


\section{Glabella tap phenomenon}

This reflex, originally described by Overend ${ }^{16}$ has been studied clinically and electrophysiologically by several investigators (Pearce et al, ${ }^{1}$ review). The reflex has two components, an initial proprioceptive myotatic monosynaptic reflex and a later multisynaptic nociceptive reflex. The initial myotatic response has a short latency with no latency fluctuation, a low threshold, fixed amplitude and is synchronous. The second response has a shorter latency and a higher amplitude. Normally this response habituates showing increasing latency and decreasing amplitude. Both components are exaggerated in Parkinsonism and also characteristic is the absence of habituation of the nociceptive reflex. In hemispherical disease only the proprioceptive response is affected. Dopaminergic nigrostriatal pathways play a role in suppressing the nociceptive part of the reflex, and the lack of habituation of the nociceptive portion in Parkinsonism suggests that the primitive glabella tap response has been released from the inhibitory influence of the striatum (or loss of dopamine inhibition). Klawans and Goodwin ${ }^{17}$ and Klawans and Paulson ${ }^{15}$ have shown a decrease in occurrence of glabella tap reflex following levodopa treatment, which can be attributed to the action of this drug on one of the specific pathways. On the other hand, the failure of levodopa to reverse the reflex in cases with organic dementia sustains the hypothesis that the exaggerated reflex may also result from diffuse cortical disease.

This observation is outside the scope of the present investigation. Our study is not longitudinal, and nearly all our patients were being treated already with levodopa or a combination therapy. This levodopa reversal of the glabella reflex could explain our rather small percentages as opposed to those of Klawans and Paulson..$^{15}$ It is, however, not evident from their paper whether or for what period these patients were on medication. A surprising finding was the large incidence (approximately $1 / 3$ ) in patients without signs of basal ganglia affection. Pearce ${ }^{12}$ studied patients with Alzheimer's disease and found percentages lower than ours. Also $1 / 3$ of our normal population had a positive glabella tap response. To the best of our knowledge, only two papers mention the incidence in a normal population, Klawans and Paulson" "probably very common" and Pearce $e t$ $a l,{ }^{1}$ who found one out of 23 subjects. This is a point of some interest. As Pearce et al' point out, regarding a positive glabella reflex as a non specific sign common to any destructive cerebral lesion fails to explain the remarkable correlation with Parkinson's disease. On the other hand, to regard a positive glabella tap response as a release phenomenon reflecting a disturbance of diffuse cerebral or frontal lobe function does not explain the equal incidence we have demonstrated in patients with intracerebral and with extracerebral or no disease.

In our evaluation of the severity of the glabella tap response, we noted an increase in the Parkinsonian group. We have, however, not made any attempt to evaluate the severity of the underlying disease, nor to compare it to our grading of the glabella tap response. To our knowledge, this has not been studied by others. On comparing the two subgroups a significant difference was found ( $p<0.05), 68$ of the 90 positive findings being in the Parkinsonian subgroup.

From our data we may conclude, that although the glabella tap response is common in basal ganglia disorders, it is also so common in intracranial disease with no obvious signs of involvement of the basal ganglia and in individuals with no signs of intracranial affection, that one can hardly lay any significance in its mere presence as an isolated phenomenon.

\section{References}

${ }^{1}$ Pearce J, Aziz H, Gallagher JC. Primitive reflex activity in primary and symptomatic Parkinsonism. $J$ Neurol Neurosurg Psychiatry 1968;31:501-8.

${ }^{2}$ Dalby MA. The diagnostic value of the palmo-mental reflex. Acta Neurol Scand 1970;46:601-8.

${ }^{3}$ Garland HG. Parkinsonism. Br Med J 1952;1:153-5.

${ }^{4}$ Webster DD. Critical analysis of the disability in Parkinson's disease. Modern Treatment 1968;5:257-82.

${ }^{5}$ Gossman MD, Jacobs L. Three primitive reflexes in Parkinsonism patients. Neurology (Minneap) 1980; 30:189-92.

${ }^{6}$ Blake JR, Kunkle EC. The palmomental reflex. Arch Neurol Psychiatry 1951;65:337-45.

${ }^{7}$ Jacobs L, Gossman MD. Three primitive reflexes in normal adults. Neurology (Minneap) 1980;30:184-8.

${ }^{8}$ Jenkyn LR, Walsh DB, Culver CM, Reeves AG. Clinical signs in diffuse cerebral dysfunction. $J$ Neurol Neurosurg Psychiatry 1977;40:956-66.

${ }^{9}$ Berkwitz NJ. Quantitative studies on the human muscle tonus II an analysis of eighty-two normal and pathological cases. Arch Neurol Psychiatry 1932;28:603-22.

${ }^{10}$ Webster DD. Dynamic measurements of rigidity, strength and tremor in Parkinson patients before and after destruction of mesial globus pallidus. Neurology (Minneap) 1960;10:157-63.

${ }^{11}$ Schwab RS, Chafetz ME, Walker S. Control of two simultaneous voluntary motor acts in normals and in Parkinsonism. Arch Neurol Psychiatry 1954;72:591-8.

${ }_{12}$ Pearce J. The extrapyramidal Disorder of Alzheimer's disease. Eur Neurol 1974;12:94-103.

${ }^{13}$ Paulson G, Gottlieb G. Development reflexes: the reappearance of foetal and neonatal reflexes in aged patients. Brain 1968;91:37-52.

${ }^{14}$ Reis DJ. The palmomental reflex. Arch Neurol 1961;4:486-98.

${ }^{15}$ Klawans HL, Paulson GW. Primitive Reflexes in Park- 
insonism. Confinia Neurol 1971;33:25-32.

${ }^{16}$ Overend W. Preliminary note on a new cranial reflex. Lancet 1896;1:619.
${ }^{17}$ Klawans HL, Goodwin JA. Reversal of the glabellar reflex in Parkinsonism by 1-dopa. J Neurol Neurosurg Psychiatry 1969;32:423-7. 